\title{
UNDECIDABLE THEORIES IN STATIONARY LOGIC
}

\author{
DETLEF SEESE, PETER TUSCHIK AND MARTIN WEESE ${ }^{1}$
}

\begin{abstract}
It is shown that the theories of the following classes become undecidable in stationary logic: Well orderings with one unary predicate, linear orderings, and Boolean algebras. This is done by interpreting the theory of symmetric reflexive graphs.
\end{abstract}

1. Introduction. This paper establishes that the $L(a a)$-theories of well orderings with a distinguished subset (WoP), linear orderings (Lin) and Boolean algebras ( $\mathrm{Ba})$ are all undecidable. This result is of interest since some decidability results (e.g., Abelian groups, reals [4], and ordinals [7] extend from $L$ to $L(Q)$ and then to $L(a a)$ ). It is known that the $L(Q)$-theories of Lin, Ba [2] and WoP (can be shown similar to the decidability of the $L(Q)$-theory of $\operatorname{Lin}$ ) are decidable. Also the $L(a a)$-theory of $\omega_{1}$ with a distinguished subset is decidable. This is a special case of the decidability of the monadic theory of $\omega_{1}[3]$.

2. Definitions and notations. We shall implicitly assume throughout the paper that the structures we consider are uncountable. For a model $\mathfrak{M}$ for $L$ its universe is denoted by $|\mathfrak{M}|$. Let $P_{1}(\mathfrak{M})$ denote the set of all countable subsets of $|\mathfrak{M}|$. A set $X \subseteq P_{1}(\mathfrak{M})$ is unbounded, if every $s_{0} \in P_{1}(\mathfrak{M})$ is a subset of some $s \in X . X$ is closed, if the union of each increasing sequence $s_{0} \subseteq s_{1} \subseteq \cdots \subseteq s_{n} \subseteq \cdots$ of elements of $X$ is again an element of $X$. A set $X$ is $c u b$, if it is closed and unbounded. $\mathscr{F}(\mathfrak{M})$ denotes the filter on $P_{1}(\mathfrak{M})$ generated by all cub sets. We define

$$
\mathfrak{M} \vDash \operatorname{aa} s \varphi(s) \quad \text { iff } \quad\left\{s \in P_{1}(\mathfrak{M}): \mathfrak{M} \vDash \varphi(s)\right\} \in \mathscr{F}(\mathfrak{M})
$$

If $\mathfrak{M} \vDash$ aa $s \varphi(s)$, we say that property $\varphi(s)$ holds for almost all countable $s$.

We assume that the reader is familiar with [1]. In that paper it is shown that the quantifier $Q_{1}$, read "there exist uncountably many", is definable in $L(a a)$ by the formula

$$
\text { stat } s \exists x(\varphi(x) \wedge \neg s(x)) .
$$

Thus $L\left(Q_{1}\right)$ is a sublogic of $L(a a)$.

Received by the editors February 26, 1980 and, in revised form, December 23, 1980.

1980 Mathematics Subject Classification. Primary 03C75; Secondary 03B25, 03C80.

Key words and phrases. Stationary logic, undecidability, linear ordering, Boolean algebra.

${ }^{1}$ We wish to thank the referee for his many valuable remarks. He simplified the interpretation of graphs as well as the proof of Theorem 3, and he carried out the proof of Theorem 6. 
Let $T$ be a theory, $\mathfrak{M} \vDash T$ and $\varphi\left(x_{1}, \ldots, x_{n}\right), \psi(s)$ formulas of the corresponding $L(a a)$-language for $T$. Then we set

$$
\begin{aligned}
\varphi^{\mathfrak{R}} & =\left\{\vec{a} \in|\mathfrak{M}|^{n}: \mathfrak{M} \vDash \varphi(\vec{a})\right\} ; \\
\psi^{\mathfrak{M}} & =\left\{s \in P_{1}(\mathfrak{M}): \mathfrak{M} \vDash \psi(s)\right\} .
\end{aligned}
$$

If $T$ is the elementary theory of some class of structures, then $T(a a)$ denotes the corresponding $L(a a)$-theory of the same class of models.

3. Preliminaries. Let $\mathrm{Gr}$ be the theory of infinite symmetric reflexive graphs. In order to show the undecidability of any theory $T$ it is enough to show that $\mathrm{Gr}$ is interpretable in $T$, i.e. there are formulas $\varphi_{0}(x), \varphi_{1}(x, y)$ and $\psi(x, y)$ such that

for each countable symmetric graph $\mathbb{S}=\langle\omega, R\rangle$ there is a model $\mathfrak{M} \vDash T$ such that $\psi^{\mathfrak{M}}$ is a congruence relation on $\left\langle\varphi_{0}^{\mathfrak{N}}, \varphi_{1}^{\mathfrak{R}}\right\rangle$ and $\left\langle\varphi_{0}^{\mathfrak{R}}, \varphi_{1}^{\mathfrak{M}}\right\rangle / \psi^{\mathfrak{M}} \simeq \mathbb{B}$.

Here we use the fact that the theory of infinite symmetric reflexive graphs is hereditarily undecidable. Let $T^{*}$ be $T$ together with a sentence $\rho$ which says of a model $\mathfrak{M} \vDash T$, that $\left\langle\varphi_{0}^{\mathfrak{M}}, \varphi_{1}^{\mathfrak{M}}\right\rangle / \psi^{\mathfrak{M}}$ is a symmetric reflexive graph. So $T^{*}$ and hence $T$ is undecidable.

A proof of the following well-known theorem can be found in [6]:

TheOREM 1 (UlAm). There is a set $\left\{S_{\alpha}: \alpha<\omega_{1}\right\}$ of pairwise disjoint stationary subsets of $\omega_{1}$.

4. Undecidability proofs. We set

$$
\begin{aligned}
\sigma(s, x, y) \equiv & \forall z(s(z) \wedge z<x \rightarrow z \leqslant y) \\
& \wedge \forall z(z<y \rightarrow \exists u(s(u) \wedge z<u \wedge u<y \wedge u<x)) .
\end{aligned}
$$

Thus, if $\mathfrak{M}$ is an ordering, $s \subseteq|\mathfrak{M}|$ and $a, b \in|\mathfrak{M}|$, and $\mathfrak{M} \vDash \sigma(s, a, b)$, then $b=\sup \{c \in|\mathfrak{M}|: s(c) \wedge c<a\}$.

Lemma 2. Let $A, B \subseteq \omega_{1}, \alpha, \beta, \gamma$ ordinals with $\alpha \neq \beta, \omega_{1}(\alpha+1), \omega_{1}(\beta+1)<\gamma$, $P=\left\{\omega_{1} \cdot \alpha+\delta: \delta \in A\right\} \cup\left\{\omega_{1} \cdot \beta+\delta: \delta \in B\right\}, \mathfrak{M}=\langle\gamma,\langle, P\rangle$. Then

$$
\text { stat } s \exists x y\left(\sigma\left(s, \omega_{1}(\alpha+1), x\right) \wedge \sigma\left(s, \omega_{1}(\beta+1), y\right) \wedge P(x) \wedge P(y)\right)
$$

iff $A \cap B$ is stationary.

Proof. For $\mathfrak{X} \in P_{1}(\mathfrak{M})$ let $\mathfrak{X}^{*}=\left\{s \in \mathfrak{X}\right.$ : for all $\delta<\omega_{1}, \omega_{1} \cdot \alpha+\delta \in s$ iff $\omega_{1} \cdot \beta$ $+\delta \in s\}$. Let

$$
\varphi(s) \equiv \exists x y\left(\sigma\left(s, \omega_{1}(\alpha+1), x\right) \wedge \sigma\left(s, \omega_{1}(\beta+1), y\right) \wedge P(x) \wedge P(y)\right) .
$$

Assume $\mathfrak{M} \vDash$ stat $s \varphi(s)$. Let $C \subseteq \omega_{1}$. We set

$$
\mathfrak{X}_{C}=\left\{\begin{array}{l}
s \in P_{1}(\mathfrak{M}): \text { there is } \delta \in C \text { with } \\
\sigma\left(s, \omega_{1}(\alpha+1), \omega_{1} \cdot \alpha+\delta\right) \text { and } \\
\sigma\left(s, \omega_{1}(\beta+1), \omega_{1} \cdot \beta+\delta\right)
\end{array}\right\} .
$$


If $C$ is closed unbounded, then $\mathfrak{X}_{C} \in \mathscr{F}(\mathfrak{M})$. Assume $A \cap B$ is not stationary. Then there is a closed unbounded subset $C$ of $\omega_{1}$ with $C \cap(A \cap B)=\varnothing$. Then $\mathfrak{X}_{C} \in$ $\mathscr{F}(\mathfrak{M})$ but for no $s \in \mathfrak{X}_{C}, \mathfrak{M} \vDash \varphi(s)$. Thus $\mathfrak{M} \vDash \neg$ stat $s \varphi(s)$. Assume on the other hand that $A \cap B$ is stationary. Let $\mathfrak{X} \in \mathscr{F}(\mathfrak{M})$. We set

$$
\begin{array}{r}
\mathfrak{X}^{*}=\left\{s \in \mathfrak{X}: \text { there is } \delta<\omega_{1} \text { with } \sigma\left(s, \omega_{1}(\alpha+1), \omega_{1} \cdot \alpha+\delta\right)\right. \\
\text { and } \left.\sigma\left(s, \omega_{1}(\beta+1), \omega_{1} \cdot \beta+\delta\right)\right\} .
\end{array}
$$

Then $\mathfrak{X}^{*} \in \mathscr{F}(\mathfrak{M})$. We set

$$
X=\left\{\delta<\omega_{1}: \text { there is } s \in \mathfrak{X}^{*} \text { with } \sigma\left(s, \omega_{1}(\alpha+1), \omega_{1} \cdot \alpha+\delta\right)\right\} .
$$

Then $X$ is a closed unbounded subset of $\omega_{1}$ and thus there is $\delta \in X \cap A \cap B$. But then there is $s \in \mathfrak{X}^{*}$ with $\mathfrak{M} \vDash \varphi(s)$ and thus $\mathfrak{M} \vDash \operatorname{stat} s \varphi(s)$.

THEOREM 3. WoP $(a a)$ is undecidable.

Proof. We set

$$
\begin{aligned}
\varphi_{0}(x) & \equiv \exists y(y<x) \wedge \forall z\left(z<x \rightarrow Q_{1} u(z<u<x)\right) \\
\varphi_{1}(x, y) & \equiv \varphi_{0}(x) \wedge \varphi_{0}(y) \wedge \text { stat } s \exists z u(\sigma(s, x, z) \wedge \sigma(s, y, u) \wedge P(z) \wedge P(u)) ; \\
\psi(x, y) & \equiv x=y .
\end{aligned}
$$

Let $\mathfrak{S}=\langle\omega, R\rangle$ be a countable symmetric reflexive graph. Let $\left\{S_{n}: n<\omega\right\}$ be a set of pairwise disjoint subsets of $\omega_{1}$ and let $h: \omega \times \omega \rightarrow \omega$ be a 1-1 function. For $k<\omega$ let

$$
A_{k}=\cup\left\{S_{h(k, i)}:(k, i) \in R\right\} \cup \cup\left\{S_{h(i, k)}:(k, i) \in R\right\} .
$$

Then each $A_{k}$ is a stationary set and $A_{k} \cap A_{i}$ is stationary iff $(k, i) \in R$. We set

$$
P=\bigcup_{k<\omega}\left\{\omega_{1} \cdot k+\alpha: \alpha \in A_{k}\right\}, \quad \mathfrak{M}=\left\langle\omega_{1} \cdot \omega,\langle, P\rangle .\right.
$$

Then Lemma 2 implies immediately that

$$
\left\langle\varphi_{0}^{\mathfrak{R}}, \varphi_{1}^{\mathfrak{R}}\right\rangle \cong \mathbb{S} \text {. }
$$

$\mathfrak{M}$ is an $\boldsymbol{\aleph}_{1}$-like dense linear ordering if $\operatorname{card}(|\mathfrak{M}|)=\boldsymbol{\aleph}_{1}$ and for each $a \in|\mathfrak{M}|$, the set $\{b \in|\mathfrak{M}|: b<a\}$ forms an order which is isomorphic to $\eta$ or $1+\eta$. For each $A \subseteq \omega_{1}$ let $\Phi(A)$ be the $\aleph_{1}$-like dense linear ordering $1+\eta+\Sigma_{\alpha<\omega_{1}} \tau_{\alpha}$ where

$$
\tau_{\alpha}= \begin{cases}1+\eta & \text { for } \alpha \in A \\ \eta & \text { otherwise }\end{cases}
$$

A proof of the following lemma can be found in [5]:

LEMMA 4 (CONWAY). For each $\aleph_{1}$-like dense linear ordering $\mathfrak{M}$ with first element there exists $A \subseteq \omega_{1}$ with $\Phi(A) \cong \mathfrak{M}$. For $A, B \subseteq \omega_{1}, \Phi(A) \cong \Phi(B)$ iff there is some closed unbounded set $C \subseteq \omega_{1}$ with $A \cap C=B \cap C$.

We use this lemma to show

THEOREM 5. Lin $(a a)$ is undecidable. 
Proof. Let \&S and $A_{k}(k<\omega)$ be as in the proof of Theorem 3. Let $\mathfrak{M}=$ $\sum_{i<\omega} \Phi\left(A_{i}\right)$ and let $a_{i}$ be the least element of $\Phi\left(A_{i+1}\right)$. Let

$$
\varphi_{0}(x) \equiv \exists y(y<x) \wedge \forall y\left(y<x \rightarrow Q_{1} z(y<z \wedge z<x)\right) .
$$

Then $\varphi_{0}^{\mathfrak{P}}=\left\{a_{i}: i<\omega\right\}$. Let

$$
\varphi_{1}(x, y) \equiv \operatorname{stat} s \exists w z(\sigma(s, x, w) \wedge \sigma(s, y, z)) .
$$

As in Lemma 2 we have $\mathfrak{M} \vDash \varphi_{1}\left[a_{i}, a_{k}\right]$ iff $A_{i} \cap A_{k}$ is stationary. (Note: for all cubs $C \subseteq \omega_{1},\left\{s: s=\Sigma_{i<\omega}\left(1+\eta+\Sigma_{\beta<\alpha} \tau_{\beta}^{i}\right)\right.$ and $\left.\alpha \in C\right\}$ is a cub in $M$.) Let

$$
\psi(x, y) \equiv x=y \text {. }
$$

We can finish the proof as in Theorem 3.

We shall show in the following that $\mathrm{Ba}(a a)$ is undecidable, thus answering a question of Eklof and Mekler [4]. We set

$$
\chi(x) \equiv Q_{1} y(y \leqslant x) \wedge \forall y\left(y \leqslant x \rightarrow \neg Q_{1} z(z \leqslant y) \vee \neg Q_{1} z(z \leqslant y \cap x)\right) .
$$

Let $\mathfrak{B}$ be a Boolean algebra, $a \in|\mathfrak{B}|$. If $\mathfrak{B} \vDash \chi[a]$, $a$ is called $\aleph_{1}$-atom. For any $a \in|\mathfrak{B}|, \mathfrak{B}[a]$ denotes the Boolean algebra consisting of all elements of $\mathfrak{B}$ which are less than or equal to $a$. Let $\mathfrak{M}$ be a linear ordering with first element. $\mathfrak{\Im}(\mathfrak{M})$ denotes the Boolean algebra generated by all left-closed right-open intervals of $\mathfrak{M}$. Let

$$
\begin{aligned}
\rho(s, x, y) \equiv & \chi(x) \wedge \forall z(s(z) \wedge z<x \wedge \neg \chi(z) \rightarrow z \leqslant y) \\
& \wedge \forall z(\forall u(s(u) \wedge u<x \wedge \neg \chi(u) \rightarrow u \leqslant z) \rightarrow y \leqslant z) .
\end{aligned}
$$

That means, if $\mathfrak{M} \vDash \rho[s, a, b]$, then $a$ is an $\aleph_{1}$-atom and $b$ is the supremum of all elements of $s$ which are less than $a$, and are not $\boldsymbol{\kappa}_{1}$-atoms.

THEOREM 6. $\mathrm{Ba}(a a)$ is undecidable.

Proof. Let $\mathbb{S}$ and $A_{k}(k<\omega)$ be as in the proof of Theorem 3. Let $\mathfrak{B}=$ $\mathfrak{\Im}\left(\sum_{i<\omega} \Phi\left(A_{i}\right)\right)$ and let $a_{i}$ be the least element of $\Phi\left(A_{i+1}\right)$. So $\mathfrak{B} \vDash \chi[b]$ iff $b=\left[a, a_{i}\right)$ $\cup b_{1} \cup \cdots \cup b_{n}$ where each $b_{k}$ is a countable interval and $a_{i-1} \leqslant a<a_{i}$ (where $a_{-1}$ is the least element of $\left.\Phi\left(A_{0}\right)\right)$. Let

$$
\varphi_{0}(x) \equiv \chi(x) ; \quad \varphi_{1}(x ; y) \equiv \text { stat } s \exists u v(\rho(s, x, u) \wedge \rho(s, y, v)) .
$$

If $b=\left[a, a_{i}\right) \cup b_{1} \cup \cdots \cup b_{n}$ and $b^{\prime}=\left[a^{\prime}, a_{j}\right) \cup b_{1}^{\prime} \cup \cdots \cup b_{m}^{\prime}$ are $\aleph_{1}$-atoms, then $\mathfrak{B} \vDash \varphi_{1}\left[b, b^{\prime}\right]$ iff $A_{i} \cap A_{j}$ is stationary. Let

$$
\psi(x, y) \equiv \chi(x) \wedge \chi(y) \wedge \chi(x \cap y) .
$$

With $b, b^{\prime}$ as above, $\mathfrak{B} \vDash \psi\left[b, b^{\prime}\right]$ iff $a_{i}=a_{j}$. Thus it is immediately seen that

$$
\left\langle\varphi_{0}^{\mathfrak{B}}, \varphi_{1}^{\mathfrak{B}}\right\rangle / \psi^{\mathfrak{B}} \cong \mathfrak{S} \text {. }
$$

\section{REFERENCES}

1. J. Barwise, M. Kaufmann and M. Makkai, Stationary logic, Ann. Math. Logic 13 (1978), 171-224.

2. A. Baudisch, D. Seese, H.-P. Tuschik and M. Weese, Decidability and generalized quantifiers, Akademie-Verlag, Berlin, 1980.

3. J. R. Büchi, The monadic second order theory of $\omega_{1}$, Lecture Notes in Math., vol. 328, Springer, Berlin and New York, 1973, pp. 1-127. 
4. P. Eklof and A. Mekler, Stationary logic of finitely determined structures, Ann. Math. Logic 17 (1980), 227-269.

5. J. Hutchinson, Order types of ordinals in models of set theory, J. Symbolic Logic 41 (1976), 489-502.

6. K. Kunen, Combinatorics, in Handbook of Mathematical Logic (J. Barwise, editor), North-Holland, Amsterdam, 1977.

7. D. Seese, Stationary logic and ordinals, Trans. Amer. Math. Soc. 263 (1981), 111-124.

AdW Der DDR, ZIMM, 108 Berlin, German Democratic Republic (Current address of Detlef Seese)

Sektion Mathematik, Humboldt-Universitat, 1086 Berlin, PSF 1297, German Democratic REPUbịIC (Current address of Peter Tuschik and Martin Weese) 